\title{
GENETIC PARAMETERS FOR CHRONIC PROGRESSIVE LYMPHEDEMA IN BELGIAN DRAUGHT HORSES
}

K. DE KEYSER ${ }^{1}$, S. JANSSENS ${ }^{1, *}$, M. OOSTERLINCK ${ }^{2}$, F. GASTHUYS ${ }^{2} \&$ N. BUYS ${ }^{1}$

${ }^{1} \mathrm{KU}$ Leuven Department of Biosystems, Livestock Genetics, 3001 Leuven, Belgium

2UGent Department of Surgery \& Anaesthesiology of Domestic Animals, Faculty of Veterinary Medicine, 9820 Ghent, Belgium

\section{INTRODUCTION \& AIM}

Belgian draught horses are susceptible to chronic progressive lymphedema (CPL). The main cause most likely is a failure of the lymphatic system of skin and subcutis, resulting in excessive protein rich interstitial fluid (lymphedema). Clinical symptoms are skin anomalies and deformation, mainly restricted to the lower limbs. Distinct symptoms are mostly present from 3 years, hence they are often missed in younger horses. With disease progression, the increasing disability frequently justifies early euthanasia in older horses, even from 6 years. A genetic background for this condition is suggested and as disease prevalence in Belgian draught horses approaches 70\%, urgent measures are needed. The aim was to estimate genetic parameters for CPL in a Belgian draught horse population in order to reduce CPL-occurrence by genetic selection.
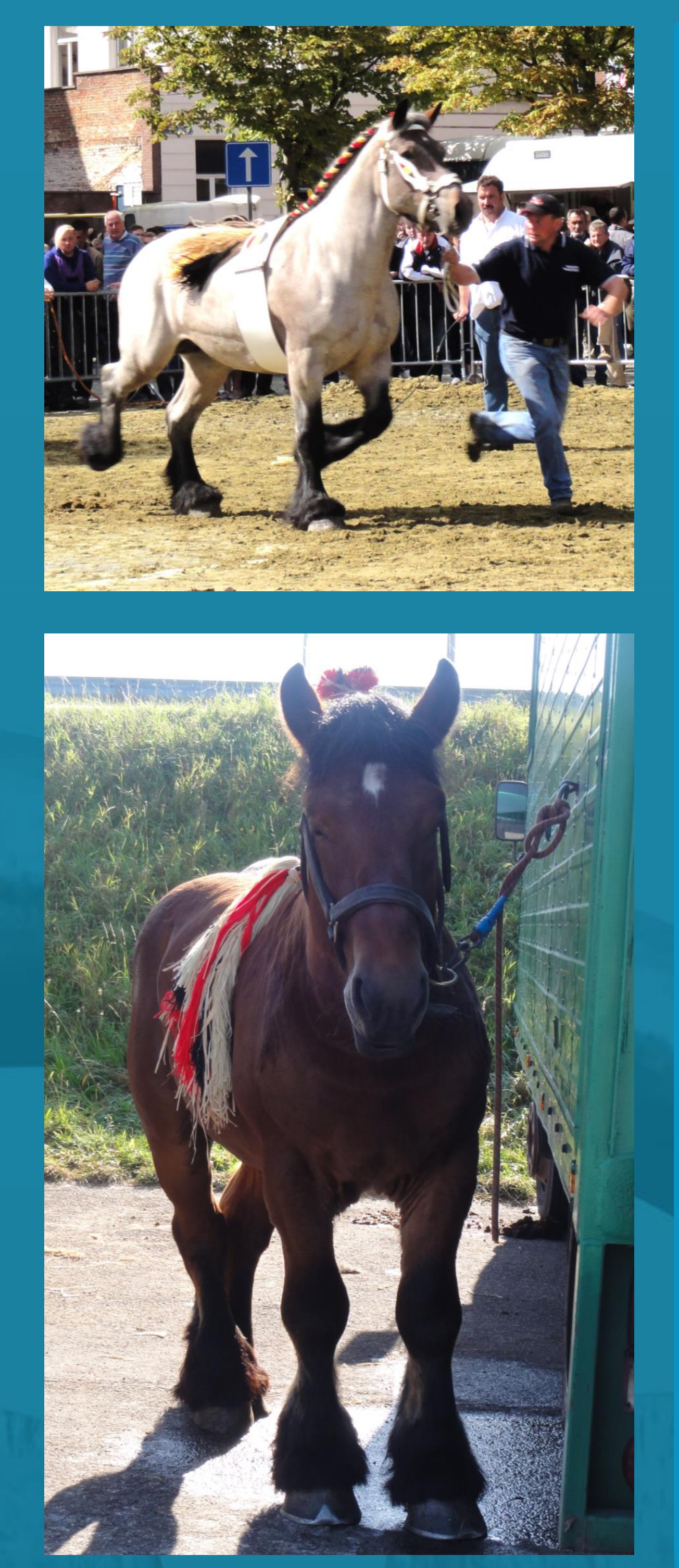

\section{MATERIALS \& METHODS}

Trait

- Sum of clinical scores 4 limbs => CPL-

score/horse

Log transformation CPL-score $=>\log C P L$

\section{Datasets}

- Data_all => all horses $(\mathrm{n}=982)$

Data_ $\geq 3 y r=>$ horses $\geq 3$ years $(n=456)$

- Selected pedigree $(n=1820)$ : sampled horses originating from 186 sires and 542 dams with an average number of ancestors $=25$

\section{Exploratory analysis of variance}

- Phenotpyic correlation $\left(r_{\text {ph }}\right)$

- ANOVA linear model (SAS9.3)

\section{Estimation of genetic parameters}

Heritabilities $\left(h^{2}\right)$ and genetic correlation $\left(r_{q}\right)$ (VCE6)

Breeding values (EBV) (VCE4)

Fixed= age, gender, coat color - Random = date of recording

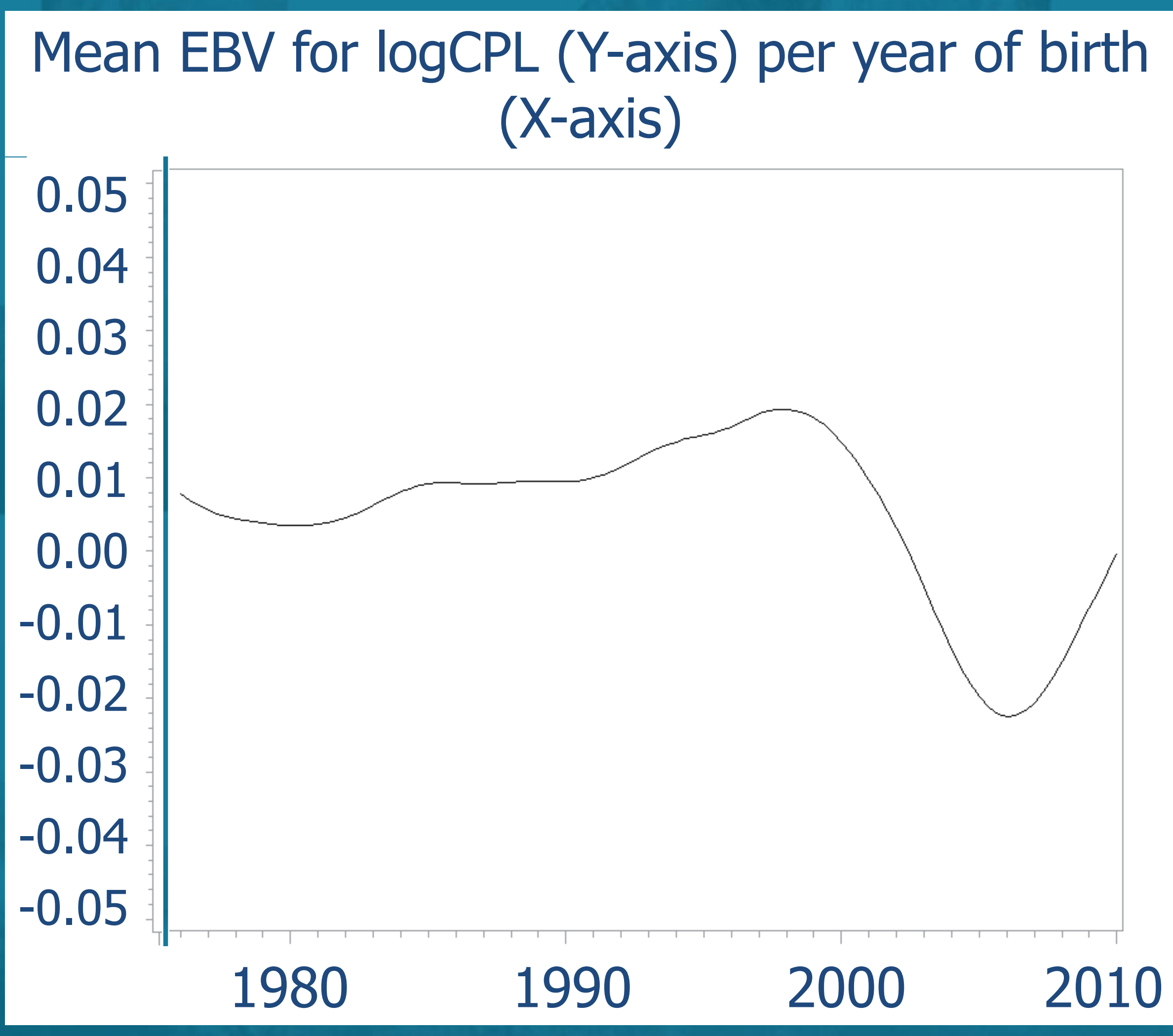

\section{RESULTS}

Exploratory analysis: logCPL data_all

\begin{tabular}{lcccr}
\hline Parameter & DF & Type III SS & F-value & Pr $>$ F \\
\hline Age*gender & 2 & 1.84 & 4.77 & 0.001 \\
\hline Age $*$ gender & 2 & 14.60 & 4.46 & $<0.001$ \\
\hline Coat color & 4 & 38.77 & 201.33 & $<0.001$ \\
\hline Date of recording & 34 & 12.48 & 64.79 & $<0.001$ \\
\hline
\end{tabular}

Exploratory analysis: logCPL data_ $\geq 3 y r$

\begin{tabular}{|c|c|c|c|c|}
\hline Parameter & DF & Type III SS & F-value & $\operatorname{Pr}>F$ \\
\hline Age*gender & 2 & 2.89 & 5.92 & $<0.001$ \\
\hline Age $^{2 *}$ gender & 2 & 16.32 & 4.61 & $<0.001$ \\
\hline Coat color & $\overline{4}$ & $\overline{7.43}$ & $\overline{20.41}$ & $<0.001$ \\
\hline Date of recordi & 29 & 3.17 & 13.00 & $<0.001$ \\
\hline
\end{tabular}
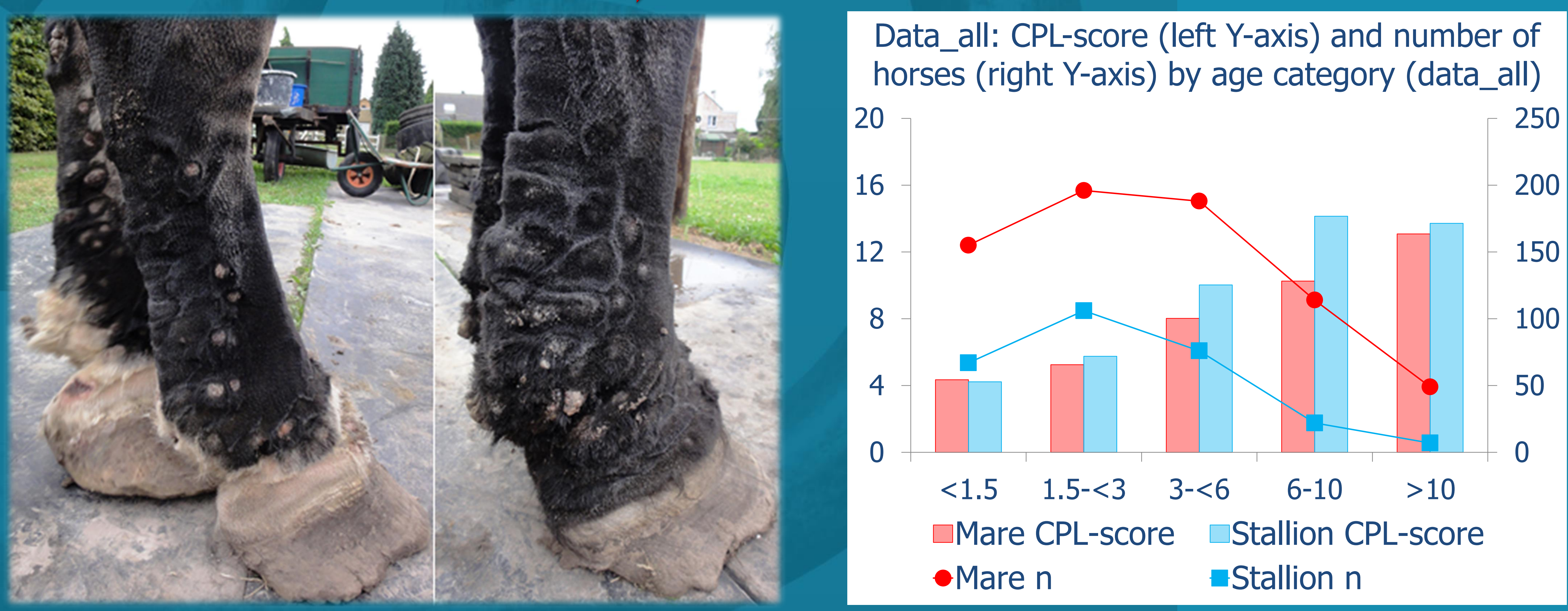

Estimation of phenotypic \& genetic parameters

\begin{tabular}{|c|c|c|c|}
\hline Dataset & & Estimate & $\mathrm{SE}$ \\
\hline \multirow{4}{*}{ Data_all } & $h^{2} \log C P L$ & 0.24 & 0.06 \\
\hline & random date of recording & 0.15 & 0.04 \\
\hline & $r_{n h}$ fore-hindlimbs & 0.85 & \\
\hline & $r_{g}$ fore-hindlimbs & 0.96 & 0.02 \\
\hline \multirow{4}{*}{ Data_>3yr } & $\mathrm{h}^{2} \log \mathrm{CPL}$ & 0.46 & 0.09 \\
\hline & random date of recording & 0.22 & 0.06 \\
\hline & $r_{p h}$ fore-hindlimbs & 0.88 & \\
\hline & $r_{0}$ fore-hindlimbs & 0.98 & 0.03 \\
\hline
\end{tabular}

\section{CONCLUSION}

- CPL-scores increase with age: at a faster rate for stallions as compared to mares

- Horses with coat color bay and black show significantly higher CPL-scores as compared to bay roan, black roan and chestnut horses

- Date of recording CPL-score is an important random factor

- Analysis on data_ $\geq 3 y r$

$=$ more certainty about CPL-status

$=$ higher heritability coefficient

- Phenotypic and genetic correlations between fore- and hindlimbs are high, sampling only forelimbs is advised (easier and safer)

- Although some variation between 2000 and 2010, EBV's for logCPL show no significant trend per year of birth
250
200
150
100
50
0 